\title{
Optimization of Ultrasound Information Imaging Algorithm in Cardiovascular Disease Based on Image Enhancement
}

\author{
Yongfu Shao, ${ }^{1}$ Jue Wu, ${ }^{1}$ Hongping Ou, ${ }^{1}$ Min Pei, ${ }^{1}$ Li Liu, ${ }^{1}$ Ali Akbar Movassagh, ${ }^{2}$ \\ Ashutosh Sharma $\left(\mathbb{D},{ }^{3}\right.$ Gaurav Dhiman, ${ }^{4}$ Mehdi Gheisari $\mathbb{D}^{5},{ }^{5}$ and Alia Asheralieva ${ }^{6}$ \\ ${ }^{1}$ Department of Ultrasonography, Xiangyang Central Hospital, Affiliated Hospital of Hubei University of Arts and Science, \\ Xiangyang 441021, China \\ ${ }^{2}$ Department of Medical Physics and Biomedical Engineering, School of Medicine, Tehran University of Medical Sciences, \\ Tehran, Iran \\ ${ }^{3}$ Institute of Computer Technology and Information Security, Southern Federal University, Rostov-on-Don, Russia \\ ${ }^{4}$ Department of Computer Science, Government Bikram College of Commerce, Patiala, India \\ ${ }^{5}$ ITMO university, Saint Petersburg, Russia \\ ${ }^{6}$ Department of Computer Science and Engineering, Southern University of Science and Technology, Shenzhen, China
}

Correspondence should be addressed to Mehdi Gheisari; mehdi.gheisari61@gmail.com

Received 6 January 2021; Revised 30 January 2021; Accepted 8 February 2021; Published 25 March 2021

Academic Editor: Essam Houssein

Copyright (c) 2021 Yongfu Shao et al. This is an open access article distributed under the Creative Commons Attribution License, which permits unrestricted use, distribution, and reproduction in any medium, provided the original work is properly cited.

To improve the interpretability or perception of information in images for human viewers is the main goal of image enhancement. Aiming at the problem that image edges are difficult to determine due to artefacts, plaques, and vascular branches in cardiovascular ultrasound, an edge ultrasound imaging detection algorithm based on spatial-frequency-domain image enhancement is proposed to improve the clarity of ultrasound images. Firstly, this paper uses the space-frequency-domain enhancement algorithm to enhance the image. This algorithm overcomes the problem of low contrast of conventional algorithms. The enhanced data matrix is used as the cost matrix, and then, the heuristic image search method is used to search the image of the cost matrix. The results show that the use of spatial-frequency-domain image ultrasound imaging algorithm can improve the contrast and sharpness of ultrasound images of cardiovascular disease, which can make the middle edge of the image clearer, the detection accuracy rate is increased to $92.76 \%$, and the ultrasound of cardiovascular disease is improved. The edge of the image gets accuracy. The paper confirms that the ultrasound imaging algorithm based on spatial-frequency-domain image enhancement is worthy of application in clinical ultrasound image processing. The performance of the proposed technique is $32.54 \%, 75.30 \%$, $21.19 \%, 21.26 \%$, and $11.10 \%$ better than the existing technique in terms of edge energy, detail energy, sharpness, contrast, and information entropy, respectively.

\section{Introduction}

Intravascular ultrasound (IVUS), a combination of noninvasive ultrasound technology and invasive catheter technology, is increasingly used in the diagnosis and treatment of coronary artery disease. It uses a miniature ultrasonic transducer mounted on the tip of the cardiac catheter to transmit and receive high-frequency ultrasound signals in the blood vessel and display the cutoff image of the blood vessel in real time, which can clearly show the thickness of the wall structure and the size and shape of the lumen and can identify calcification, fibrosis and lipid pool, and other diseases [1]. The midouter edge is an important basis for the diagnosis and quantitative analysis of cardiovascular disease and is also an important feature in IVUS image processing and analysis. Accurate and rapid detection of the midouter edge is of great importance. As a diagnosis tool, intravascular ultrasound (IVUS) imaging is a technique which is based on the catheter, and it provides the image of the lumen and wall 
morphology information and renders an artery 2D crosssectional image $[2,3]$. Intravascular ultrasound (IVUS) imaging, as a diagnosis tool, is a catheter-based technique which renders a $2 \mathrm{D}$ cross-sectional image of arteries and provides information concerning the lumen and wall morphology [4]. The role of IVUS imaging is very critical to determine artery stent specifications [5]. Two distinct borders are generally used to characterize the arteries. The lumen-wall interface is represented by the lumen border, and the boundary between the media and adventitia is represented by the mediaadventitia border [6]. The lumen and mediaadventitia borders of a general IVUS image are shown in Figure 1.

Among the existing IVUS images, the outer-membrane edge-detection algorithm, the most widely used, is based on the active contour model, also known as Snake $[7,8]$. The basic idea of the traditional active contour model is to find a parameterized contour curve in the image with a weighted sum of internal energy and external energy as a minimum value. However, the model has a large dependence on the initial position and poor anti-interference ability and is not easy to converge at the boundary depression.

Compared with the abovementioned defects of the snake algorithm, the graph search algorithm based on the dynamic programming idea has the advantages of overcoming noise interference, not manually drawing the initial contour, being unaffected by the initial conditions and model parameters, and adding heuristic information to guide the search process. It can greatly improve search efficiency and correct rate. Therefore, heuristic map search is widely used in IVUS image processing. As early as 1995, Milan proposed a graph search method based on the least cost algorithm for edge extraction of IVUS images, but its cost matrix is designed for IVUS images obtained by early low-frequency ultrasound probe scanning, which is not suitable for current highfrequency ultrasound probes. The resulting IVUS image with high resolution, high noise characteristics is scanned. In response to this problem, the author used edge energy information and edge direction information to construct a cost matrix to search for the midouter edge, but the energy and direction of the plaque and artefact edges are very similar to the midadventives edge $[9,10]$. It is easy to be mistaken for the midouter edge.

From the abovementioned analysis, it can be seen that whether the correct edge can be found by using the graph search method depends on whether the cost matrix can reflect the characteristics of the target edge, and the image enhancement can directly improve the contrast information of the middle film, the outer film, and the interface thereof. The target edge feature is highlighted, so this paper uses the image enhancement method to construct the cost matrix. Image enhancement refers to the extension of an image to a specific need. It can be divided into spatial-domain enhancement and frequency-domain enhancement by scope. Frequency-domain enhancement is the processing of the spectral components of an image to enhance the image. In the frequency-domain image enhancement algorithm, the directional filter group-based enhancement algorithm can accurately extract the direction information of the image,

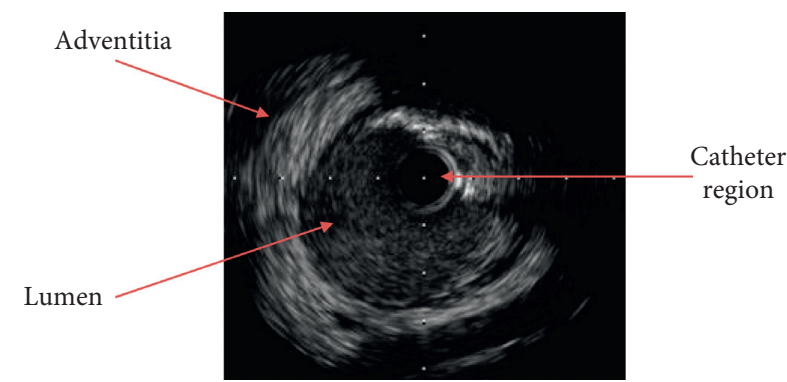

FIgURe 1: General IVUS image.

which is often applied to some image enhancements with obvious directional features, but the enhanced image has contrast and clarity. There are problems such as reduced degrees and partial loss of features. Therefore, the image enhancement processing of a single domain each has certain defects.

Based on the abovementioned problems, an image enhancement algorithm combining the spatial domain and frequency domain is proposed. The algorithm based on neighbourhood information is used to combine the directional filter group (DFB) enhancement in the frequency domain and the histogram equalization (HE) enhancement in the spatial domain. The IVUS image is enhanced to overcome the defects caused by single domain enhancement while highlighting the region of interest. On this basis, the enhanced image data is used as a cost matrix to perform dynamic plan image search to obtain the midouter film edge. Figure 2 is a block diagram of the algorithm of this paper. The experimental results show that the proposed algorithm can overcome the problems of noise, artefacts, and plaque interference and accurately extract the midfilm edge of the IVUS image.

This paper is structured as follows. The review of the literature followed by the contribution of the paper is detailed in Section 2. Details of image enhancement algorithms are discussed in Section 3. Discussion of experimental results obtained and comparative analysis with $2 \mathrm{D}$ compression techniques is presented in Section 4, and Section 5 concludes the paper.

\section{Literature Review}

Many researchers have worked on the image enhancement of the intravascular ultrasound image in cardiovascular disease. Zhou et al. proposed a novel technique for optimal contrast enhancement of a medical image [11]. For the enhancement and to highlight the image information and to detail the World Cup Optimization (WCO) algorithm design, the gamma correction method is the main idea of the author. A suitable gamma correction method enhances the image coefficient which is dependent upon the correct selection. Khalil et al. provide an overview of the state-of-theart image registration methods to impart valuable resource for studying the existing methods [12]. New methods are also developed and evaluated for cardiac image registration. Perperidis et al. proposed dynamic histogram-based intensity mapping (DHBIM), a novel method in which 


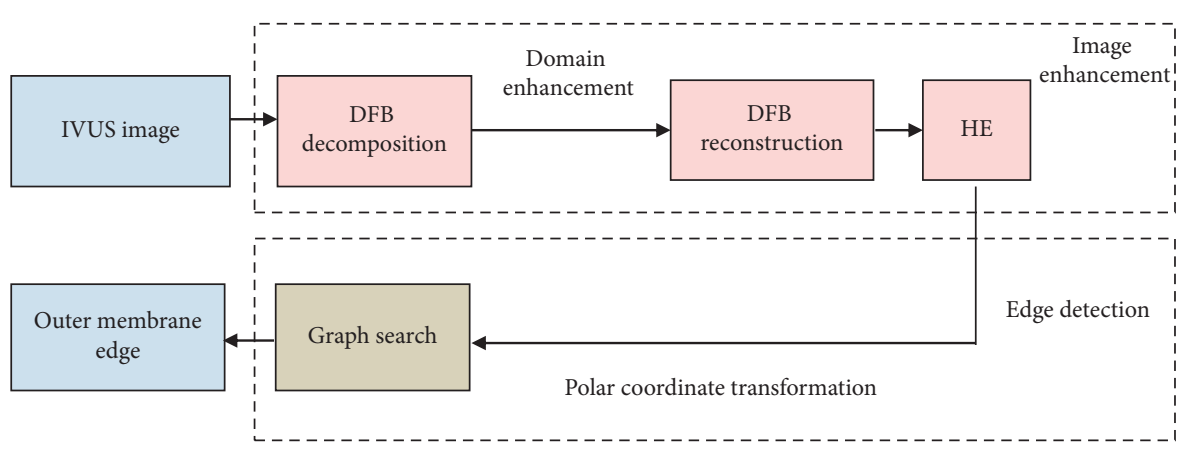

Figure 2: Algorithm frame structure.

temporal variations are employed in cumulative histograms of cardiac ultrasound images, to enhance the image contrast [13]. To compensate for noise and speckle, DHBIM is combined with spatial compounding. The proposed technique is assessed qualitatively and introduces $120.9 \%$ increase in tissue/chamber detectability, improving the overall repeatability of clinical measurements by $17 \%$. Anam et al. proposed a modified Perona-Malik diffusion (PMD) filter for the enhancement of the coronary plaque boundary, and the conditions peculiar to an intravascular ultrasound (IVUS) image are also considered [14]. Generally, an IVUS image is used for the diagnosis of acute coronary syndrome (ACS). The proposed technique reduces the speckle noise and also enhances the coronary plaque boundary. The coronary plaque boundaries are detected successfully by applying the Takagi-Sugeno fuzzy model. Huang and Zeng reviewed on how to design an interactive system with appropriate processing algorithms remain missing that result in the lack of systematic understanding of the relevant technology [15]. The exhaustive previous and latest work is reviewed on designing a real-time or near real-time $3 \mathrm{D}$ ultrasound imaging system. Different techniques such as the data acquisition techniques, reconstruction algorithms, volume rendering methods, and clinical applications are also presented. Akkus et al. proposed several methods for analyzing intraplaque neovascularisation (IPN) perfusion and structure. Six different quantitative parameters-IPN surface area (IPNSA), IPN surface ratio (IPNSR), plaque mean intensity, plaque-to-lumen enhancement ratio, mean plaque contrast percentage, and number of microvessels (MVN)are obtained and compared [16]. Basij et al. describe an automated algorithm for shadow region detection and enhancement in (IVUS) images [17]. An adaptive threshold method contour approach is used for border detection and image enhancement algorithm including histogram analysis for the shadow regions improvement. The author proposed Otsu threshold for calcification plaque segmentation and for shadow region separation of the image and the active contours without edge method. Loizou and Pattichis proposed despeckle filters based on texture analysis, image quality evaluation metrics, and visual evaluation by medical experts [18]. These filters remove the Despeckle noise, and it will improve the visual observation quality. Senior discussed about the cography (SE) to improve visualization of the endocardial contrast agents [19]. To improve accuracy of wall motion assessment and to reduce the number of uninterpretable images, these agents are widely used. $\mathrm{Nu}$ merous single and multicenter studies have proved that myocardial contrast perfusion stress echocardiography is now a clinical tool for the detection of CAD. Smith et al. proposed ultrasound contrast agents (UCAs) to enhance cardiovascular ultrasound imaging [20, 21]. Adverse biological effects have occurred after administration of UCAs, and the ApoE-/- mouse model of atherosclerosis is proposed to characterize the effects of ultrasound and UCAs on atherosclerosis and plasma biomarkers.

2.1. Contribution. Due to artefacts, plaques, and vascular branches in cardiovascular ultrasound, it is difficult to determine the image edges, and there is a suitable method reported in the literature for this purpose. In this paper, to improve the clarity of ultrasound images, an edge ultrasound imaging detection algorithm based on spatial frequency domain image enhancement is proposed. Also, to determine the image edges efficiently, a midadventure edge detection algorithm for cardiovascular ultrasound images combined with air-frequency-domain enhancement is proposed. The algorithm effectively combines the directional filtering in the frequency domain with the neighbourhood enhancement and histogram enhancement of the spatial domain and overcomes the defects of the spatial image enhancement algorithm, the fuzzy image detail, and the frequency-domain enhancement algorithm to reduce the image contrast and then the enhancement result as a cost.

\section{Methodology Used}

In this section, different algorithms used for the enhancement of ultrasound images in cardiac diseases are discussed. The proposed ultrasound image enhancement algorithm is detailed briefly in this section.

\subsection{Air-Frequency Domain Combined with Image Enhancement.} The image enhancement algorithm combined with the airfrequency domain combines the image enhancement algorithm based on the directional filter bank with the image enhancement algorithm based on the histogram equalization to overcome the defects caused by the single-domain 
enhancement and achieve the enhancement of the image target edge [22-24].

3.1.1. Directional Filter Bank. As an effective directional feature information extraction tool, the directional filter group (DFB) can accurately acquire the direction information of an image, so it is widely used in the direction texture enhancement of images. The first two-stage decomposition structure of the directional filter bank is shown in Figure 3(a), where $H_{0}, H_{1}$ is a sector filter, the black area represents the ideal pass band of the filter, and $Q_{0}, Q_{1}$ is the 5 -type sampling matrix shown in equation (1). The corresponding sampling grid is shown in Figure 4. The image is decomposed to obtain a frequency division in the four directions as shown in Figure 3(b), and the reconstructed portion is a corresponding dual operation.

$$
\begin{aligned}
& Q_{0}=\left[\begin{array}{cc}
1 & -1 \\
1 & 1
\end{array}\right], \\
& Q_{1}=\left[\begin{array}{cc}
1 & 1 \\
-1 & 1
\end{array}\right] .
\end{aligned}
$$

The directional filter bank can effectively extract 2D information by splitting the subbands of the image, which researchers often use for image enhancement. Some researchers have used the directional filter bank for the enhancement of fingerprint images. The process directly performs DFB decomposition on the fingerprint image and weights the subband coefficients by estimating the directional energy information of the subband image. Finally, the image enhancement result is reconstructed.

3.2. Histogram Equalization. Histogram equalization (HE) can quickly and effectively enlarge the gravy dynamic range of the image and improve the overall visual effect of the image. It is the most important and most common algorithm in the spatial image enhancement algorithm. In the histogram equalization process, the combination of gravy levels representing high-frequency components leads to the loss of image detail information, especially for images with low gravy-valued pixels, and the equalized image grayscale will be concentrated [25]. The second half of the histogram merges too many gravy values, resulting in more detail loss; at the same time, due to the existence of multiple noises in the image, when the dynamic range of the gravy level of the image is increased, the noise of the image is magnified.

Histogram equalization is often used for enhancement of low-contrast images, especially for images with less noise and less detailed information [26, 27].

\subsection{Spatial- and Frequency-Domain-Combined Image En-} hancement Based on Neighbourhood Information. Image enhancement algorithms using a single domain, whether based on DFB-based image enhancement or HE-based image enhancement, cannot achieve better enhancement effects due to certain defects. The DFB-based enhancement enhances the direction information of the image and also reduces the contrast of the image. The HE-based enhancement can improve the brightness and contrast of the image, but blurs the image detail to amplify the noise $[28,29]$. Therefore, aiming at the characteristics of IVUS images, an enhanced algorithm combining spatial and frequency domains is proposed to improve the contrast information of the middle and outer membranes and their interfaces and highlight the midadventive edge features. The basic steps of the algorithm are as follows.

3.3.1. DFB Decomposition of IVUS Images. Performing a 2level DFB decomposition on the IVUS image $F$ will result in 4 subband images $Q_{j}(j=1,2,3,4)$. Since the direction of the main power spectrum of the image in the frequency domain is orthogonal to the direction of the texture in the spatial domain, each subband image expresses texture detail information orthogonal to the four directions as shown in Figure 3(b).

\subsubsection{Enhancing the Direction Information Expressed by} Each Subband Image Using the Method Based on Neighbourhood Information. Each subband image expresses detailed texture information in a certain direction, as shown in Figure 5. In order to avoid the loss of such information, each subband image is enhanced by a method based on neighbourhood information. It is divided into the following two steps:

(1) It is determined whether the pixel in the subband is in the bright texture area or the dark texture area. The neighborhood block of $w \times w$ size is set around each pixel in each subband. The average gray value $m_{j}(x, y)$ of all pixels in the neighborhood block represents the average brightness of all textures within the block. Therefore, when the gray value $Q_{j}(x, y)(j=1,2,3,4)$ of the central pixel of the block is greater than the neighborhood average $m_{j}(x, y)$, it is considered to be in the brighter texture region in the neighborhood; conversely, if it is smaller than the neighborhood average, it is considered to be dark in the neighborhood texture area.

(2) The pixel gradation value is adjusted according to the judgment result of step (1). In order to improve the contrast between the bright texture area and the dark texture area, that is, to make the bright area brighter and the dark area darker, a method of weighting the difference $Q_{j}(x, y)-m_{j}(x, y)$ between the gravy value of the central pixel and the mean value of the gravy in the neighbourhood block is adopted.

(3) A new gravy value $N_{j}(x, y)$ is calculated, and the gravy value of the original centre pixel is replaced with the new gravy value. When the central pixel is in the bright region, the difference between the gravy value of the point and the mean value of the gravy in the neighbourhood block is positive, the positive value is multiplied by a weight greater than 1 to obtain a larger positive value, which is replaced by the original gravy value, and the gravy value of the 


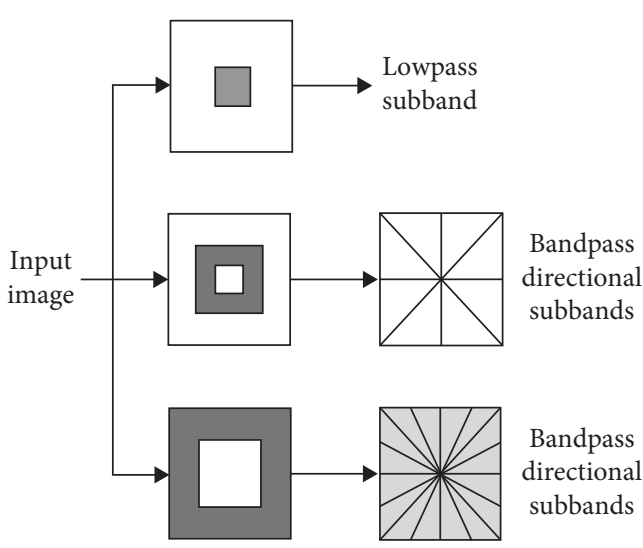

(a)

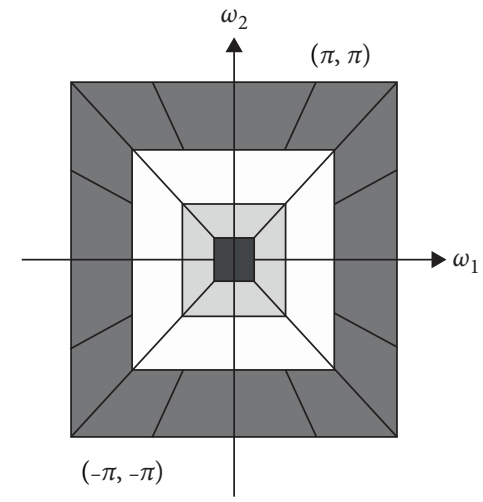

(b)

FIgURE 3: DFB implementation structure diagram and band decomposition diagram.

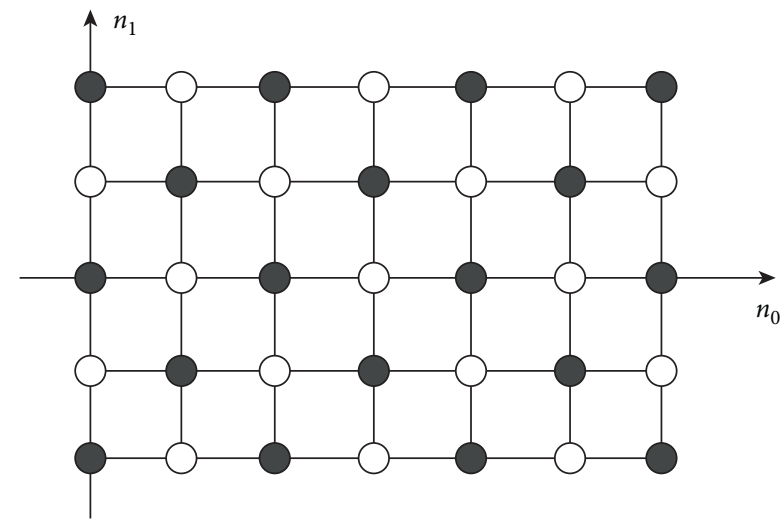

FIGURE 4: Sampling network corresponding to sampling matrix $Q_{0}, Q_{1}$.

point is improved; when the central pixel is in the dark area, the difference is negative, multiplying the negative value by a weighting value greater than 1 to obtain a smaller negative value. When it replaces the original gravy value, the gravy value of the point is lowered.

$$
N_{j}(x, y)=k\left[Q_{j}(x, y)-m_{j}(x, y)\right] .
$$

The abovementioned algorithm processes the subband image, which can improve the contrast of the middle and outer film boundaries, and it is an enhancement process for performing or gaining or attenuating the gravy value of each pixel, so that the image is enhanced while not being missed.

\subsubsection{Performing DFB Reconstruction on Each Subband Image} after Enhancement and Performing Histogram Equalization on the Reconstructed Image. DFB reconstruction is performed on each subband image $N_{j}(j=1,2,3,4)$ after enhancement. After reconstruction, the gravy level of the image $G$ is concentrated in a narrow intermediate area, and the gravy- level dynamic range is narrow, resulting in a low contrast and brightness of the entire image. In this paper, the histogram equalization algorithm is used to nonlinearly stretch the image, redistribute the pixel values, adjust the gravy level range to uniform distribution, increase the global contrast of the image, and obtain the final enhanced image $H$ [30-32].

3.4. Combined with Image Enhancement in Intravascular Ultrasound- Epicardial Edge Detection. A heuristic map search algorithm was used in the previous section to obtain the midadventives edge. The image under enhanced polar coordinates is treated as a connected graph. The gravy value of each pixel represents the cost of the nodes in the cost matrix [33-36]. The proposed algorithm is divided into two parts:

(a) Image enhancement

(b) Edge detection

The sum of the values of all nodes on a complete path is the sum of the costs of the path of all possible paths; the accumulated cost and the largest or smallest path are the optimal edges of the target. The flowchart of the proposed algorithm is shown in Figure 6.

(1) The enhanced image $H$ is converted to the image $I$ in polar coordinates. The point $\left(x_{0}, y_{0}\right)$ of the catheter centre position of the enhanced image $H$ is the origin of the polar coordinate system (determined by the IVUS imaging device, the centre of the catheter in the original image is always at the centre coordinate position of the image, so the centre point of the catheter is set to the pole point in this paper, the origin of the coordinate system); the angular direction samples $M$ points according to the sampling rate $\theta=2 \pi /(M-1)$; the radial sampling $N$ points according to the sampling rate $C$; and the information lost during the discretization and transformation is recovered by bilinear interpolation. After the polar coordinate transformation, the approximately circular closed contour becomes a 


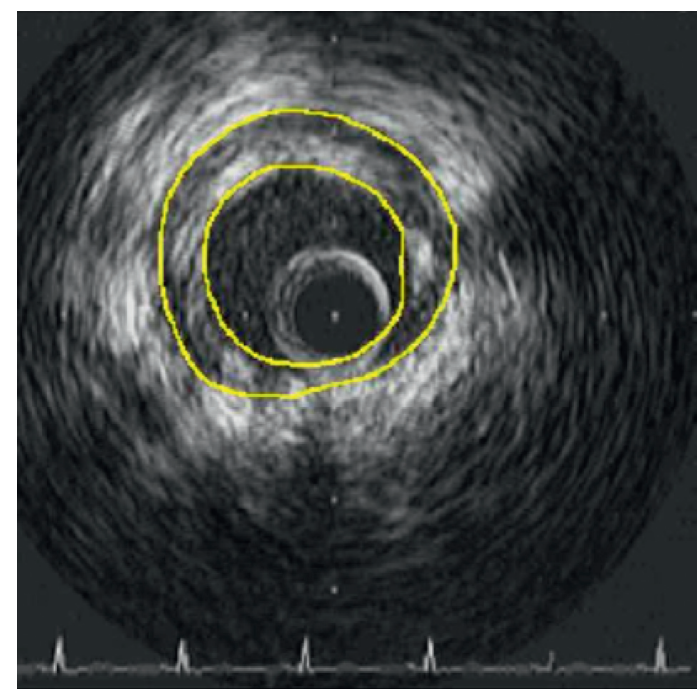

Figure 5: Image to be enhanced IVUS.

horizontal edge line, which meets the requirement of searching by column in rows of rows in a minimum cost method.

(2) The minimum cost and matrix are calculated. The first column element retains the original value, starting with the second column, looking for the minimum value in the neighbourhood of the previous column of each row element of the column. The previous column neighbourhood here refers to the 3 pixels (the first row and the last row of two pixels) adjacent to the element in the previous column.

(3) The sum of the original value of the element and the minimum value of the neighbourhood of the previous column found in step (2) is taken as the new value of the position element.

(4) Steps (2) and (3) are repeated until the element values (except the first column) at each coordinate point of the matrix are updated, thereby obtaining a minimum cost and matrix $Q$.

(5) From the cost and matrix $Q$ obtained in step (4), the minimum value from each element in the last column is found and the coordinate point position is recorded.

(6) The minimum value in the neighbourhood of the previous column of the position of the element obtained in step (5) is found, and the coordinate point position is recorded thereof.

(7) The reverse search of step (6) is repeated until the corresponding minimum value of the first column is found. The recorded coordinate point position is the minimum cost and path sought.

\section{Experimental Results and Analysis}

The effectiveness and accuracy of the proposed algorithm are verified by the image enhancement effect and the midadventive edge detection effect. The experimental data was obtained from a clinical IVUS image provided by the Leiden University Medical Centre in the Netherlands. The imaging system operates at a frequency of $40 \mathrm{MHZ}$. A 600-frame IVUS image with a size of $500 \times 500$ and a gravy level of 256 was randomly selected from the library (including 86 images of the vessel branch image and 443 images containing the plaque, containing both the vessel branch and the plaque image, 26. There were 97 images without plaque and no vascular branches), and it participated in the experiment. The experiment was implemented on the matlab2010b platform.

4.1. Enhancement Effect. The algorithm of this paper and the existing algorithm $[37,38]$ are used to enhance the extracted 600 frames, and the enhancement effect on IVUS images is analysed from both qualitative and quantitative aspects. The author believes that when calculating gradients and Laplacian operators in diffusion equations, larger-scale templates with more neighbourhood pixels are used. More accurate data can be obtained, and in order to increase the visual effect of the ultrasound image, a nonquadratic regularization factor is added to the diffusion equation. This algorithm has a better enhancement effect on ultrasound images with simple tissue and lesion structure. The experiment uses a 24-neighbor template, and the parameters are set according to empirical values. In the algorithm of this paper, the value of $k$ in equation (2) will affect the gravy distribution range of the image: if the value of $k$ is too large, the grayscale distribution of the image after the enhancement is wide, which is inconvenient for subsequent processing; if $k$ used is small, it cannot effectively improve the image contrast. The size of the neighbourhood block $w \times w$ affects the judgment of the texture area (light texture area or dark texture area) of the central pixel. In order to achieve the purpose of enhancing the edge, the side length $w$ should be larger than the width of the middle-outer film edge. Three or four texture sizes are taken. According to some sample test results statistics and clinician experience, the algorithm parameters in this paper take $k=2 ; w=15$.

Figure 7 is a result of extracting a typical IVUS original image of the presence of fibrous plaques, calcified plaques, mixed plaques, and vascular branches from the obtained results and enhanced result images using the existing algorithm and the proposed algorithm. Comparing Figure 7(a), it can be found that, for the image containing fibre plaque, the existing algorithm [37] enhances the contrast between the bright and dark areas, but the degree of blurring of the detail texture in the image increases, and the clock is visible. The interface between the small plaque and the media at 8 o'clock is not clear. The visual effect of the posterior tissue at the 2 o'clock direction is not as good as the algorithm. As can be seen from Figure $7(\mathrm{~b})$, the existing algorithm cannot enhance the calcification plaque, attenuated echo area (clock 1 to 4 o'clock); as can be seen from Figure $7(\mathrm{c})$, the existing algorithm cannot enhance the echo zone affected by mixed plaques with uneven reflection (clock 1 to 5 o'clock direction), and the interface between the small plaque and the middle membrane is blurred (clock 7 to 9 o'clock direction). However, the enhancement algorithm of this paper 


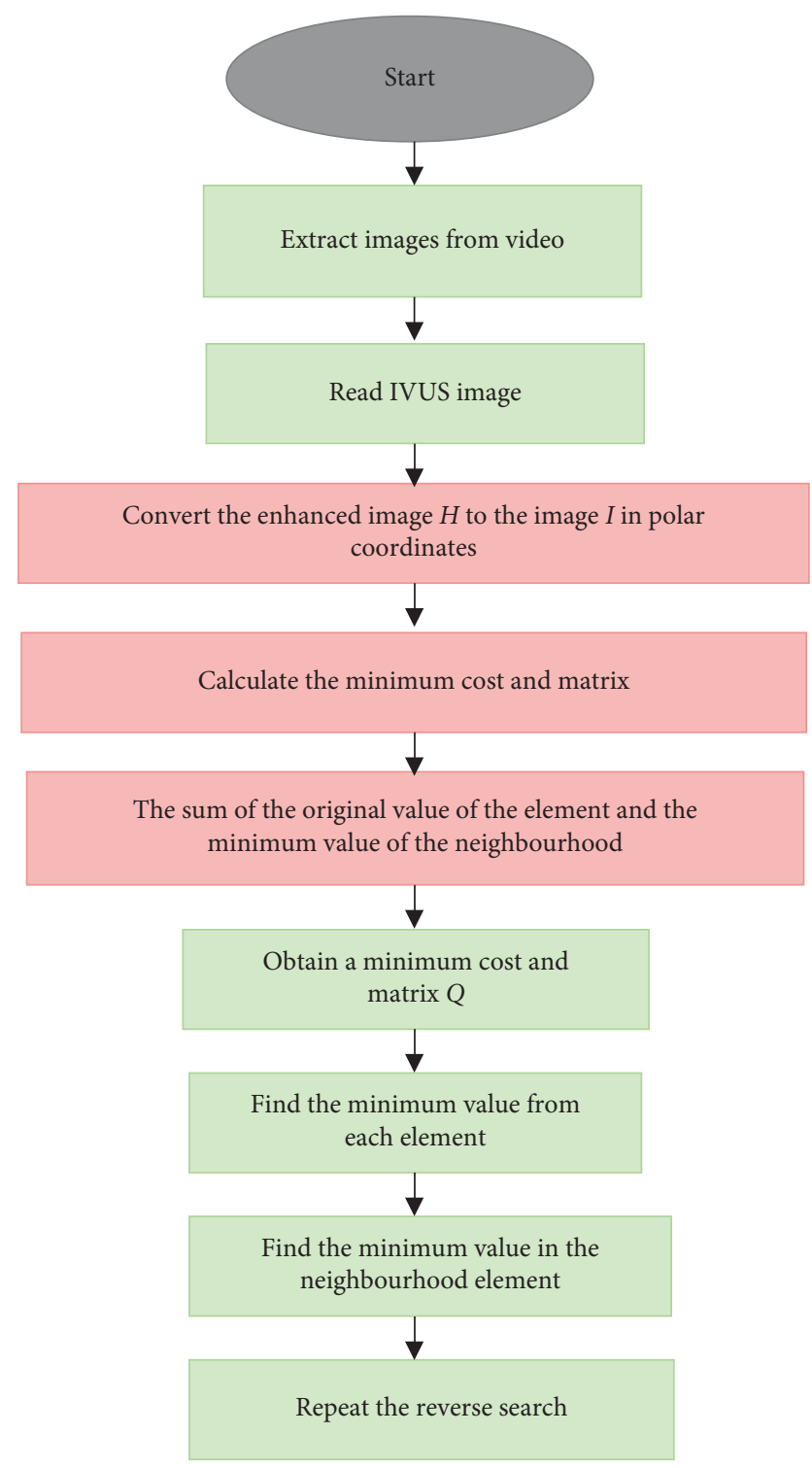

FIgURE 6: Flowchart of the proposed algorithm.

can enhance the weak echo region which is attenuated behind the calcified plaque to the naked eye, and the complex and uneven echo zone brought by the mixed plaque can be enhanced to be clearly distinguishable, which greatly reduces the complexity.

Various image enhancement parameters such as edge enhancement, detail energy, sharpness, contrast, and information entropy of the image are used to quantify the enhancement effects of the IVUS image, as shown in Table 1. Among them, the 600 image frames of images are utilized for experiment, and then, the average value was obtained.

The values of the performance parameters obtained by the proposed technique are better which shows the enhanced quality of the image.

4.2. Edge Detection Effect. For the 600 frames of images extracted, the algorithm was used to perform the midouter film edge detection on the experimental images. In order to verify the effectiveness of the proposed algorithm for edge detection, the enhancement algorithm proposed by Song and Zhang is used to replace the enhancement algorithm for comparison experiments. At the same time, to verify the accuracy of the edge detection of the whole algorithm, the algorithm as proposed by Song and Zhang 2015 is also introduced for comparison. The midadventitial edge of the doctor with clinical experience was used as the standard to evaluate the effect of the algorithm on the detection of the midadventitial edge from both qualitative and quantitative aspects. In the experiment, the whole sample was divided into three groups: an image containing a blood vessel branch, an image containing a plaque, and an image that contained neither of them. The middle-outer film edge was detected by three algorithms for each image in each group in the existing algorithm [37], and the initial contour is set near the edge of the real intimal.

The algorithm is more accurate for the detection of plaques containing plaques, calcified plaques, mixed plaques, and images containing blood vessels. It can be seen from the figure that the cost matrix obtained based on the enhanced algorithm can not only overcome the influence of fibre plaque but also can effectively enhance the complex low echo region brought by hard plaque and mixed plaque. The correct edge is detected. In addition, the midadventitial edge can be accurately detected for IVUS images with branching [26, 28].

Comparative observations show that the midouter edge obtained by the algorithm is closest to the doctor's tracing results. Table 2 is the correct rate statistics of the results for the whole experimental sample, which is introduced by the curve similarity. When the similarity between the test result and the doctor's trace edge curve is greater than 0.7 , the test result is considered correct; otherwise, it is considered that the test result is incorrect (parameter $z=2$ ).

4.3. Comparison with the State-of-the Art Techniques. The results obtained from the proposed technique are compared with the state-of-the art techniques to show the performance of the proposed technique. The image enhancement parameters such as edge enhancement, detail energy, sharpness, contrast, and information entropy of the image are compared with the existing techniques, as in Table 3. The abovementioned parameters were calculated for the original image, the enhanced image of the existing algorithm [37], and the enhanced image of the proposed algorithm, and then, the average values were obtained.

It can be seen from Table 3 that both the algorithm previously proposed by Song and Zhang and the proposed technique improve the richness and clarity of the edges (high edge energy) and the contrast of the image. However, the former enhances the detail information of the image which is not obvious (from the low detail energy enhancement and the low information entropy can be seen), and the enhancement of the image clarity is not large. The algorithm of this paper has been greatly improved in the abovementioned aspects, which shows that the algorithm improves the sharpness and contrast of the image while enhancing the edge and detail information. In addition, the algorithm in this paper causes a certain lack of information, which is because the direction filter group 

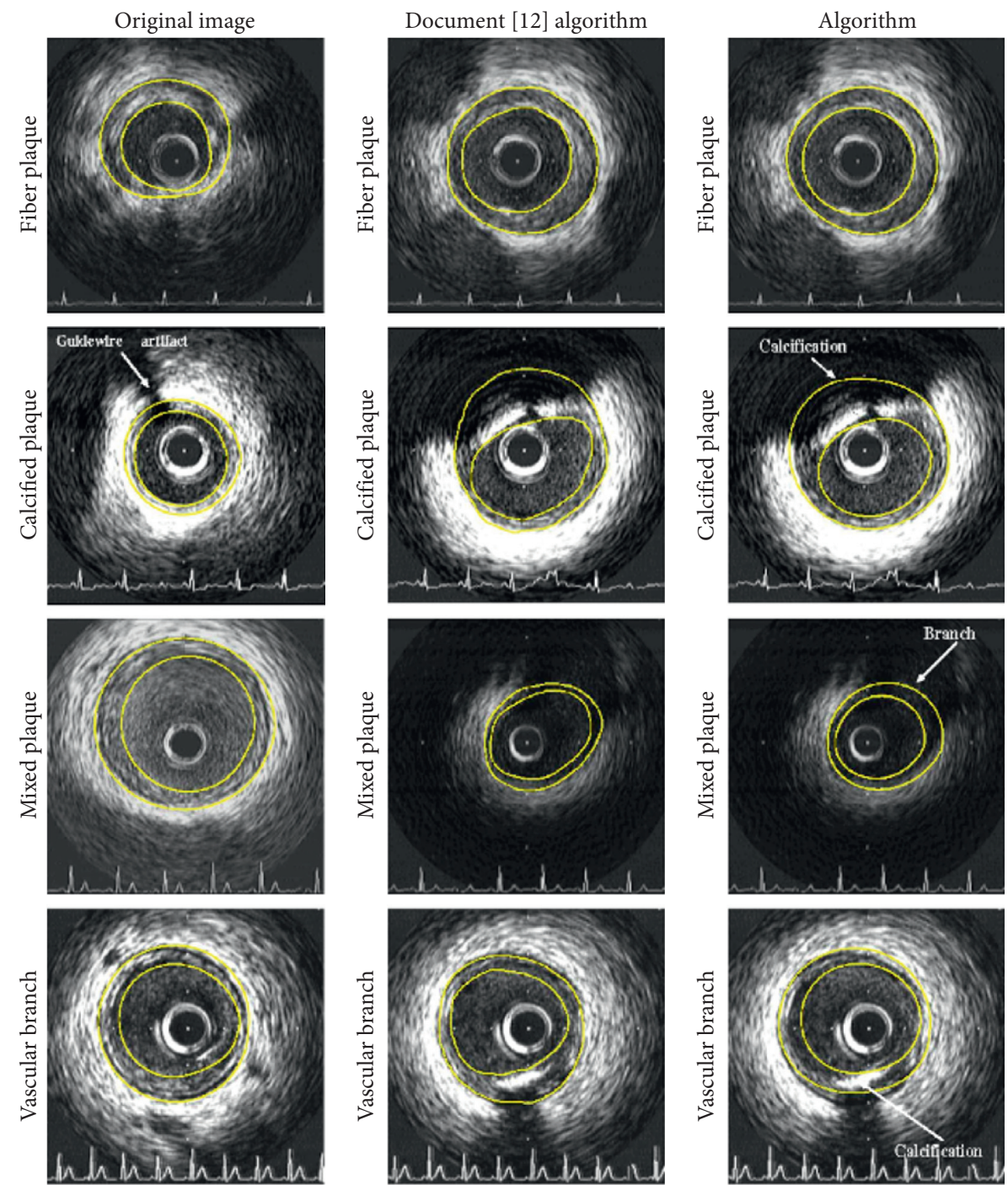

(a)

(b)

(c)

FIGURE 7: Image enhancement comparison experiment results.

TABLE 1: Average values of various image enhancement parameters.

\begin{tabular}{lcc}
\hline Parameters & Original image & Proposed algorithm \\
\hline Edge energy & 0.0035 & 0.0255 \\
Detail energy & 0.0058 & 0.0243 \\
Sharpness & 0.0715 & 0.1137 \\
Contrast & 0.0314 & 0.1326 \\
Information entropy & 6.5321 & 5.6739 \\
\hline
\end{tabular}

inevitably causes the loss of image information during the sampling and interpolation process. The values of the performance parameters of the original image, enhanced images obtained by existing technique, and the proposed technique are represented in Figure 8. The graphical representation shows that the image quality enhanced by the proposed technique is better as compared to the existing technique.

The performance of the proposed technique is compared with that of the existing technique in terms of different performance parameters, and the percentage improvement is
TABLe 2: Correct rate statistics of the results for the whole experimental sample.

\begin{tabular}{lc}
\hline Parameters & Proposed \\
\hline Plaque-containing image & 89.39 \\
Vascular branch image & 93.02 \\
No vascular branches or plaque images & 95.88 \\
Total correct rate & 92.76 \\
\hline
\end{tabular}

represented graphically in Figure 9. It is clear from the figure that the proposed technique outperforms the existing technique by $32.54 \%, 75.30 \%, 21.19 \%, 21.26 \%$, and $11.10 \%$ in terms of edge energy, detail energy, sharpness, contrast, and information entropy, respectively.

The comparison of the proposed technique is carried out with other existing image enhancement algorithms, as tabulated with Table 4. Comparative observations show that the midouter edge obtained by the algorithm is closest to the doctor's tracing results. Table 4 is the correct rate statistics of 
Table 3: Comparison with the existing technique.

\begin{tabular}{lccc}
\hline Parameters & Original image & {$[37]$} & Algorithm \\
\hline Edge energy & 0.0035 & 0.0172 & 0.0255 \\
Detail energy & 0.0058 & 0.006 & 0.0243 \\
Sharpness & 0.0715 & 0.0896 & 0.1137 \\
Contrast & 0.0314 & 0.1044 & 0.1326 \\
Information entropy & 6.5321 & 5.0436 & 5.6739 \\
\hline
\end{tabular}

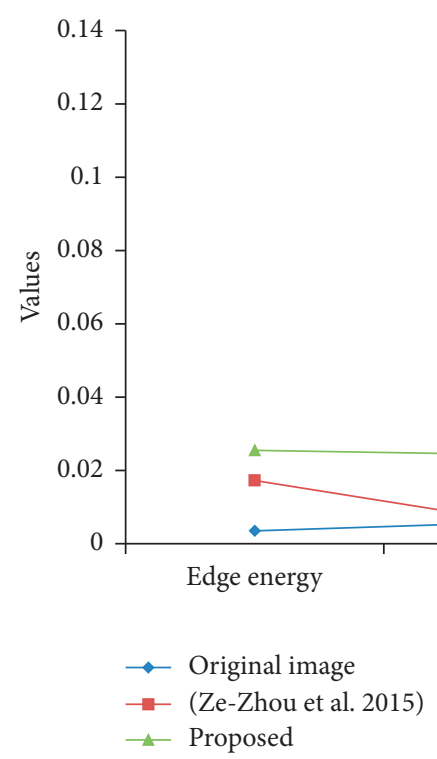

Figure 8: Performance parameters of the original image and enhanced images obtained by the existing technique and the proposed technique.

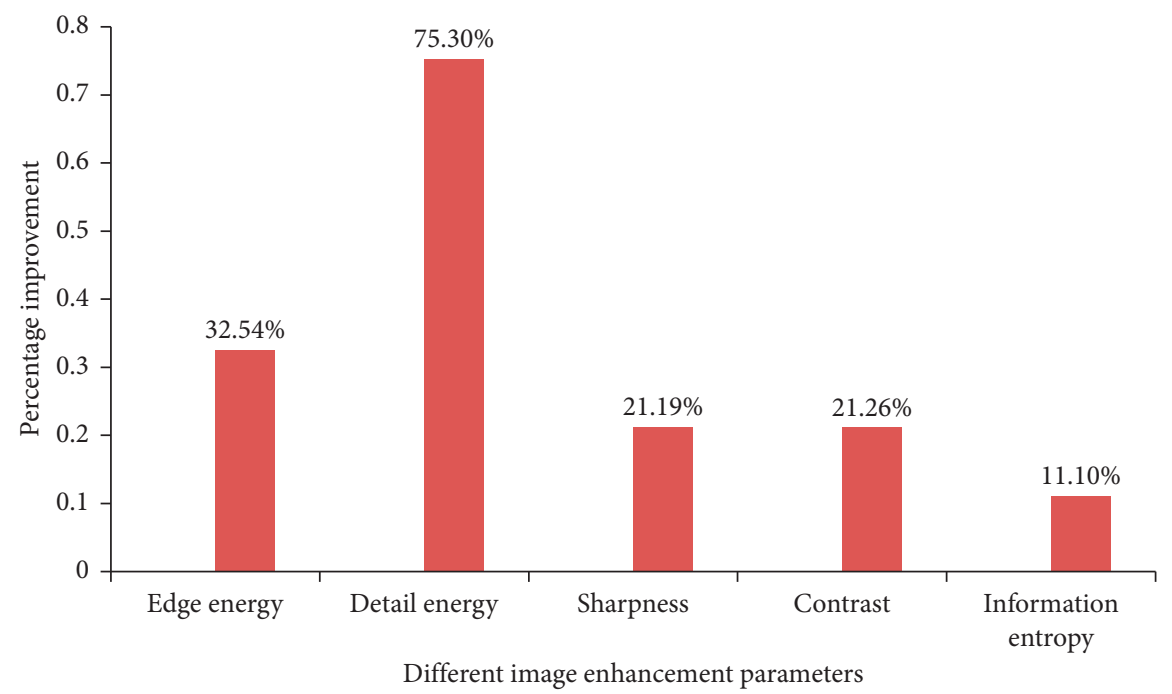

Figure 9: Percentage improvement of the proposed technique over the existing techniques.

the results of the three experimental algorithms for the whole experimental sample, which is introduced by the curve similarity: When the similarity between the test result and the doctor's trace edge curve is greater than 0.7 , the test result is considered correct; otherwise, the test result is considered incorrect (parameter $z=2$ ).
Comparative results of the proposed technique and other existing techniques are graphically represented, as shown in Figure 10. Trend of the results obtained by the proposed technique and Song and Zhang is the same, but the proposed technique performs better as cleared from Figure 10. 
TABLE 4: Comparison of the correct rate of the outer membrane detection in the three algorithms.

\begin{tabular}{lccc}
\hline & {$[37]$} & {$[20]$} & Proposed \\
\hline Plaque-containing image & 79.01 & 85.78 & 89.39 \\
Vascular branch image & 84.88 & 75.58 & 93.02 \\
No vascular branches or plaque images & 87.63 & 94.85 & 95.88 \\
Total correct rate & 83.84 & 85.4 & 92.76 \\
\hline
\end{tabular}

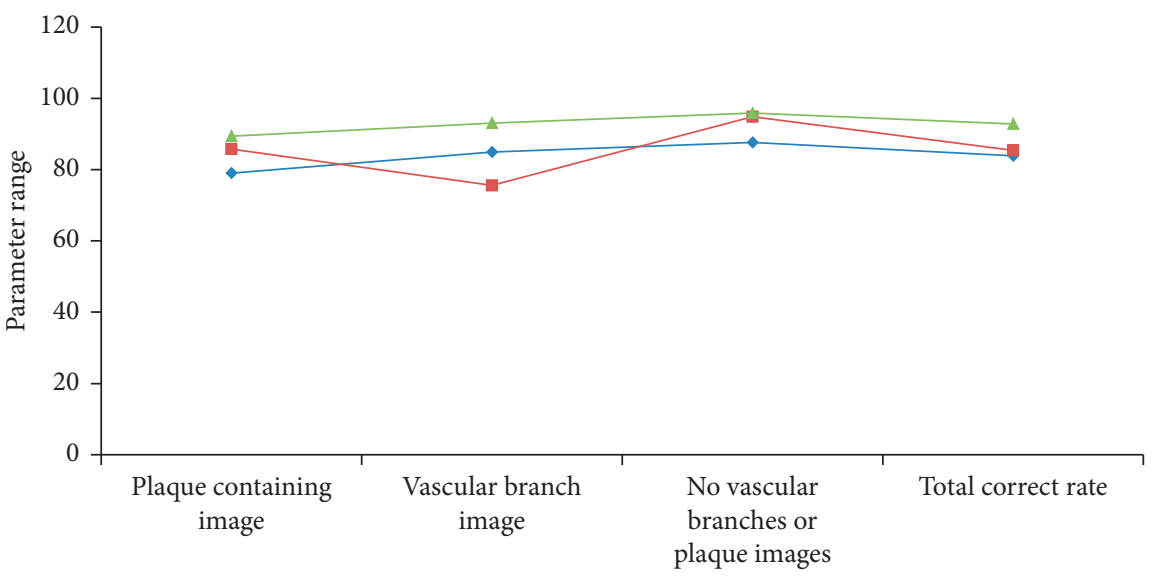

Different parameters

$\rightarrow$ (Ze-Zhou et al. 2015)

$\rightarrow$ (Smith et al. 2015)

$\triangle$ Proposed

FIgURE 10: Comparative results of the proposed technique.

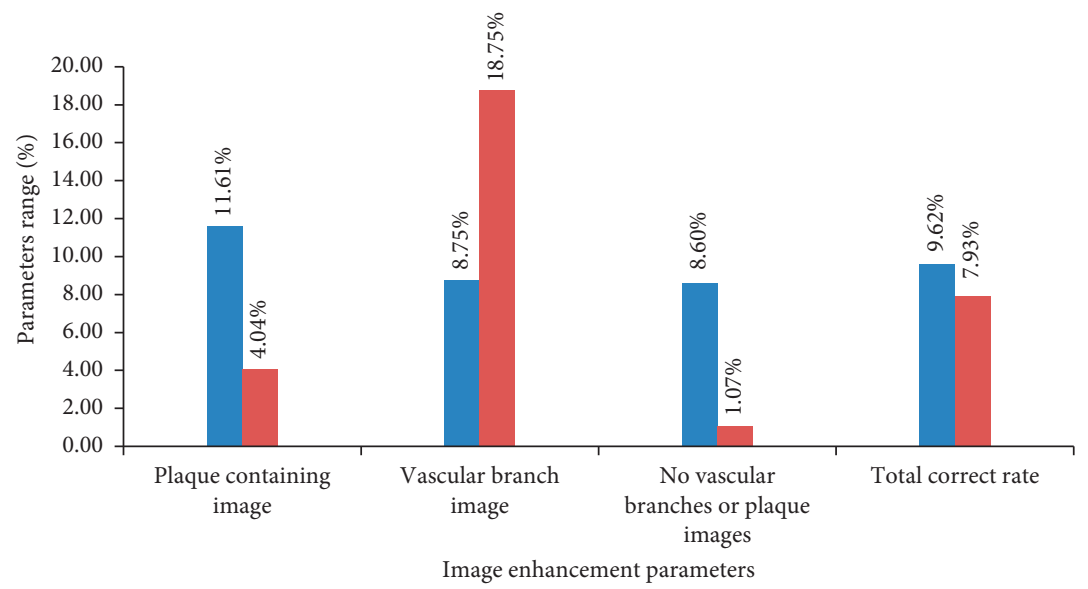

Over (Ze-Zhou et al. 2015)

- Over (Smith et al. 2015)

Figure 11: Percentage improvement of the proposed technique. 
The performance of the proposed technique is also presented in terms of percentage improvement, as shown in Figure 11.

The proposed technique outperforms the existing technique proposed by Song and Zhang by $8 \%$ to $11 \%$. The results of the proposed technique are $1 \%$ to $8 \%$ better than the existing technique that is proposed by Smith et al.. It can be seen that the researchers will apply this approach for solving the real-life complex problems also [39-58].

\section{Conclusions}

This paper proposed a midadventure edge detection algorithm for cardiovascular ultrasound images combined with air-frequency-domain enhancement. The algorithm effectively combines the directional filtering in the frequency domain with the neighbourhood enhancement and histogram enhancement of the spatial domain and overcomes the defects of the spatial image enhancement algorithm, the fuzzy image detail, and the frequency-domain enhancement algorithm to reduce the image contrast and then the enhancement result as a cost. The matrix performs a graph search to find the minimum cost and path that represent the midouter membrane edge. The experimental results show that the proposed algorithm can better overcome the influence of plaque and sound shadow on the middle-outer membrane detection and can also detect the blood vessels of some special structures without manual intervention, accuracy, and robustness. The performance of the proposed technique is $32.54 \%, 75.30 \%, 21.19 \%, 21.26 \%$, and $11.10 \%$ better than the existing technique in terms of edge energy, detail energy, sharpness, contrast, and information entropy, respectively. The proposed technique's performance is better than that of the very well-known techniques that are proposed by the Song and Zhang and Smith et al. The proposed technique is $8 \%$ to $11 \%$ better as compared to Song and Zhang's technique, and it is $1 \%$ to $8 \%$ better than the technique proposed by Smith et al..

\section{Data Availability}

The data used to support the findings of this study are included within the article.

\section{Ethical Approval}

All procedures performed in studies involving human participants were in accordance with the ethical standards of the institutional and/or national research committee and with the 1964 Helsinki Declaration and its later amendments or comparable ethical standards. This article does not contain any studies with animals performed by any of the authors.

\section{Consent}

Informed consent was obtained from all individual participants included in the study.

\section{Conflicts of Interest}

The authors have no conflicts of interest.

\section{Acknowledgments}

This work was supported in part by the National Natural Science Foundation of China under project 61950410603. This work was financially supported by the Government of the Russian Federation through the ITMO Fellowship and Professorship Program.

\section{References}

[1] C. P. Loizou and C. S. Pattichis, Despeckle Filtering for Ultrasound Imaging and Video, Volume II: Selected Applications, Second Edition, Morgan \& Claypool, San Rafael, CA, USA, pp. 1-180, 2015.

[2] X. Feng, X. Guo, and Q. Huang, "Systematic evaluation on speckle suppression methods in examination of ultrasound breast images," Applied Sciences, vol. 7, no. 1, p. 37, 2017.

[3] Q. Huang, Y. Luo, and Q. Zhang, "Breast ultrasound image segmentation: a survey," International Journal of Computer Assisted Radiology and Surgery, vol. 12, no. 3, pp. 493-507, 2017.

[4] M. Papadogiorgaki, V. Mezaris, Y. S. Chatzizisis, G. D. Giannoglou, and I. Kompatsiaris, "Image analysis techniques for automated IVUS contour detection," Ultrasound in Medicine \& Biology, vol. 34, no. 9, pp. 1482-1498, 2008.

[5] M. Schmauder, C. M. Gross, M. Firle, J. Waigand, and R. Orglmeister, "Automated detection of endovascular contours from intravascular ultrasound image sequence using a simulated annealing approach. Book of abstracts," in In Proceedings of the 8th Mediterranean Conference on Medical and Biological Engineering and Computing, Lemesos, Cyprus, June 1998.

[6] R. Sanz-Requena, D. Moratal, D. R. García-Sánchez, V. Bodí, J. J. Rieta, and J. M. Sanchis, "Automatic segmentation and 3D reconstruction of intravascular ultrasound images for a fast preliminar evaluation of vessel pathologies," Computerized Medical Imaging and Graphics, vol. 31, no. 2, pp. 71-80, 2007.

[7] J. Wu, Y. Wang, J. Yu et al., "Intelligent speckle reducing anisotropic diffusion algorithm for automated 3-D ultrasound images," Journal of the Optical Society of America A, vol. 32, no. 2, pp. 248-257, 2015.

[8] J. Du, X.-L. Mao, P.-F. Ye, and Q.-H. Huang, "Three-dimensional reconstruction and visualization of human enamel ex vivo using high-frequency ultrasound," Journal of Medical and Biological Engineering, vol. 37, no. 1, pp. 112-122, 2017.

[9] D. Tenbrinck and X. Jiang, "Image segmentation with arbitrary noise models by solving minimal surface problems," Pattern Recognition, vol. 48, no. 11, pp. 3293-3309, 2015.

[10] P. P. Rezaeiye, M. Gheisari, and E. Karbalayi, "Statistical method used for doing better corneal junction operation," Advanced Materials Research, vol. 548, pp. 762-766, 2012.

[11] Y. Zhou, C. Shi, B. Lai, and G. Jimenez, "Contrast enhancement of medical images using a new version of the world cup optimization algorithm," Quantitative Imaging in Medicine and Surgery, vol. 9, no. 9, pp. 1528-1547, 2019.

[12] A. Khalil, S. C. Ng, Y. M. Liew, and K. W. Lai, "An overview on image registration techniques for cardiac diagnosis and 
treatment," Cardiology Research and Practice, vol. 2018, Article ID 1437125, 15 pages, 2018.

[13] A. Perperidis, D. Cusack, A. White, N. McDicken, T. MacGillivray, and T. Anderson, "Dynamic enhancement of B-mode cardiac ultrasound image sequences," Ultrasound in Medicine \& Biology, vol. 43, no. 7, pp. 1533-1548, 2017.

[14] S. Anam, E. Uchino, and N. Suetake, "Coronary plaque boundary enhancement in IVUS image by using a modified perona-malik diffusion filter," International Journal of Biomedical Imaging, vol. 2014, Article ID 740627, 9 pages, 2014.

[15] Q. Huang and Z. Zeng, "A review on real-time 3D ultrasound imaging technology," BioMed Research International, vol. 2017, Article ID 6027029, 20 pages, 2017.

[16] Z. Akkus, A. Hoogi, G. Renaud et al., "New quantification methods for carotid intra-plaque neovascularization using contrast-enhanced ultrasound," Ultrasound in Medicine \& Biology, vol. 40, no. 1, pp. 25-36, 2014.

[17] M. Basij, A. Taki, and M. Yazdchi, "Automatic shadow enhancement in intra vascular ultrasound (IVUS) images," in Proceedings of the 2nd Middle East Conference on Biomedical Engineering, pp. 309-312, IEEE, Doha, Qatar, February 2014.

[18] C. P. Loizou and C. S. Pattichis, Despeckle Filtering for Ultrasound Imaging and Video, Volume I: Algorithms and Software, Second Edition, Morgan \& Claypool, San Rafael, CA, USA, pp. 1-180, 2015.

[19] R. Senior and B. N. Shah, "Myocardial contrast echocardiography for simultaneous assessment of function and perfusion in real time: a technique comes of age," Circulation, vol. 126, no. 10, pp. 1182-1184, 2012.

[20] B. W. Smith, D. G. Simpson, S. Sarwate, R. J. Miller, J. W. Erdman Jr., and W. D. O’Brien Jr., "Contrast ultrasound imaging of the aorta does not affect progression of atherosclerosis or cardiovascular biomarkers in ApoE-/-Mice," Journal of Ultrasound in Medicine, vol. 34, no. 6, pp. 11151122, 2015.

[21] X. Zhang, F. Fan, M. Gheisari, and G. Srivastava, "A novel auto-focus method for image processing using laser triangulation," in IEEE Accessvol. 7, pp. 64837-64843, 2019.

[22] S. I. Park, M. J. Smith, and R. M. Mersereau, "A new directional filter bank for image analysis and classification," in Proceedings of the 1999 IEEE International Conference on Acoustics, Speech, and Signal Processing ICASSP99 (Cat. No. 99CH36258), vol. 3, pp. 1417-1420, IEEE, Phoenix, AZ, USA, March 1999.

[23] G. Swamy and K. Balasubramaniam, "Directional filter bankbased segmentation for improved evaluation of nondestructive evaluation images," NDT \& E International, vol. 40, no. 3, pp. 250-257, 2007.

[24] R. H. Bamberger and M. J. T. Smith, "A filter bank for the directional decomposition of images: theory and design," IEEE Transactions on Signal Processing, vol. 40, no. 4, pp. 882-893, 1992.

[25] O. Patel, Y. P. Maravi, and S. Sharma, "A comparative study of histogram equalization based image enhancement techniques for brightness preservation and contrast enhancement," 2013, http://arxiv.org/abs/1311.4033.

[26] J. Yang, W. Zhong, and Z. Miao, "On the Image enhancement histogram processing," in Proceedings of the 2016 3rd International Conference on Informative and Cybernetics for Computational Social Systems (ICCSS), pp. 252-255, IEEE, Jinzhou, China, August 2016.

[27] M. Gheisari and M. Esnaashari, "A survey to face recognition algorithms: advantageous and disadvantageous," Journal
Modern Technology \& Engineering, vol. 2, no. 1, pp. 57-65, 2017.

[28] S. Maerivoet, An Introduction to Image Enhancement in the Spatial Domain, University of Antwerp, Department of Mathematics and Computer Science, Antwerp, Belgium, 2000.

[29] G. R. Sinha, "Design and implementation of image enhancement techniques in frequency domain," Ph. D. Thesis, Chhattisgarh Swami Vivekanand Technical University, Bhilai, India, 2009.

[30] M. H. Asmare, V. S. Asirvadam, and A. F. M. Hani, "Image enhancement based on contourlet transform," Signal, Image and Video Processing, vol. 9, no. 7, pp. 1679-1690, 2015.

[31] D. Liang, "Image enhancement based on the nonsubsampled contourlet transform and adaptive threshold," Acta Electronica Sinica, vol. 36, no. 3, p. 527, 2008.

[32] Y. H. Huang and D. W. Chen, "Image fuzzy enhancement algorithm based on contourlet transform domain," Multimedia Tools and Applications, vol. 79, pp. 35017-35032, 2019.

[33] A. H. Twing, J. Meyer, H. Dickens, M. N. Young, and A. Shroff, "A brief history of intracoronary imaging," Current Cardiovascular Imaging Reports, vol. 13, no. 6, p. 18, 2020.

[34] R. Lanjewar, K. Barapatre, M. Petkar, and P. Pande, "IVUS detection using image processing: a review," International Journal of Innovative Research in Computer and Communication Engineering, vol. 3, no. 3, pp. 2549-2553, 2015.

[35] J. A. Noble and D. Boukerroui, "Ultrasound image segmentation: a survey," IEEE Transactions on Medical Imaging, vol. 25, no. 8, pp. 987-1010, 2006.

[36] J. Sethuraman, J. A. Alzubi, R. Manikandan, M. Gheisari, and A. Kumar, "Eccentric methodology with optimization to unearth hidden facts of search engine result pages," Recent Patents on Computer Science, vol. 12, no. 110, 2019.

[37] Z.-Z. Song and Y. M. Zhang, "Contrast-enhanced ultrasound imaging of the vasa vasorum of carotid artery plaque," World Journal of Radiology, vol. 7, no. 6, pp. 131-133, 2015.

[38] J. A. Alzubi, A. Yaghoubi, M. Gheisari, and Y. Qin, "Improve heteroscedastic discriminant analysis by using CBP algorithm," in Proceedings of the Algorithms and Architectures for Parallel Processing ICA3PP 2018, J. Vaidya and J. Li, Eds., vol. 11335, Springer, Cham, Switzerland, Lecture Notes in Computer Science, 2018.

[39] G. Dhiman and V. Kumar, "Spotted hyena optimizer: a novel bio-inspired based metaheuristic technique for engineering applications," Advances in Engineering Software, vol. 114, pp. $48-70,2017$.

[40] G. Dhiman and V. Kumar, "Emperor penguin optimizer: a bio-inspired algorithm for engineering problems," Knowledge-Based Systems, vol. 159, pp. 20-50, 2018.

[41] G. Dhiman and V. Kumar, "Multi-objective spotted hyena optimizer: a multi-objective optimization algorithm for engineering problems," Knowledge-Based Systems, vol. 150, pp. 175-197, 2018.

[42] G. Dhiman and V. Kumar, "Seagull optimization algorithm: theory and its applications for large-scale industrial engineering problems," Knowledge-Based Systems, vol. 165, pp. 169-196, 2019.

[43] G. Dhiman and A. Kaur, "STOA: a bio-inspired based optimization algorithm for industrial engineering problems," Engineering Applications of Artificial Intelligence, vol. 82, pp. 148-174, 2019.

[44] S. Kaur, L. K. Awasthi, A. L. Sangal, and G. Dhiman, “Tunicate swarm algorithm: a new bio-inspired based metaheuristic 
paradigm for global optimization," Engineering Applications of Artificial Intelligence, vol. 90, p. 103541, 2020.

[45] G. Dhiman, "ESA: a hybrid bio-inspired metaheuristic optimization approach for engineering problems," Engineering with Computers, vol. 37, pp. 323-353, 2019.

[46] G. Dhiman and M. Garg, "MoSSE: a novel hybrid multiobjective meta-heuristic algorithm for engineering design problems," Soft Computing, vol. 24, pp. 18379-18398, 2020.

[47] G. Dhiman, K. K. Singh, A. Slowik et al., "EMoSOA: a new evolutionary multi-objective seagull optimization algorithm for global optimization," International Journal of Machine Learning and Cybernetics, vol. 12, pp. 571-596, 2020.

[48] G. Dhiman, D. Oliva, A. Kaur et al., "BEPO: a novel binary emperor penguin optimizer for automatic feature selection," Knowledge-Based Systems, vol. 211, p. 106560, 2021.

[49] G. Dhiman, K. K. Singh, M. Soni et al., "MOSOA: a new multiobjective seagull optimization algorithm," Expert Systems with Applications, vol. 167, p. 114150, 2021.

[50] H. Kaur, A. Rai, S. S. Bhatia, and G. Dhiman, "MOEPO: a novel multi-objective emperor penguin optimizer for global optimization: special application in ranking of cloud service providers," Engineering Applications of Artificial Intelligence, vol. 96, p. 104008, 2020.

[51] M. Dehghani, Z. Montazeri, A. H. Dehghani et al., "DM: dehghani method for modifying optimization algorithms," Applied Sciences, vol. 10, no. 21, p. 7683, 2020.

[52] A. Malik, P. K. Singh, A. Sharma, and R. Kumar, "An efficient architecture for the accurate detection and monitoring of an event through the sky," Computer Communications, vol. 148, pp. 115-128, 2019.

[53] A. Sharma, P. K. Singh, and Y. Kumar, "An integrated fire detection system using IoT and image processing technique for smart cities," Sustainable Cities and Society, vol. 61, p. 102332, 2020.

[54] D. Kumar, A. Sharma, R. Kumar, and N. Sharma, "Restoration of the network for next generation $(5 \mathrm{G})$ optical communication network," in Proceedings of the 2019 International Conference on Signal Processing and Communication (ICSC), pp. 64-68, IEEE, Noida, India, March 2019.

[55] A. Sharma, R. Sarishma, R. Tomar, N. Chilamkurti, and B.-G. Kim, "Blockchain based smart contracts for internet of medical things in e-healthcare," Electronics, vol. 9, no. 10, p. 1609, 2020.

[56] A. Sharma and R. Kumar, "Computation of the reliable and quickest data path for healthcare services by using servicelevel agreements and energy constraints," Arabian Journal for Science and Engineering, vol. 44, no. 11, pp. 9087-9104, 2019.

[57] C. D. Johnson, L. E. A. Montgomery, J. G. Quinn, S. M. Roe, M. T. Stewart, and E. A. Tansey, "Ultrasound imaging in teaching cardiac physiology," Advances in Physiology Education, vol. 40, no. 3, pp. 354-358, 2016.

[58] F. Pelliccia, P. Palmiero, M. Maiello, and M.-A. Losi, "Italian chapter of the international society of cardiovascular ultrasound expert consensus document on training requirements for noncardiologists using hand-carried ultrasound devices," Echocardiography, vol. 29, no. 6, pp. 745-750, 2012. 